Note

\title{
Development and Validation of Rapid Analysis Method for Multi-Class Veterinary Drugs in Livestock Products by LC-MS/MS
}

(Received March 30, 2012)

\author{
Takayuki Nakajima*, Chieko Nagano, Takeo Sasamoto, Hiroshi Hayashi, Maki Kanda, \\ Setsuko Kanai, Kazue Takeba, Yoko Matsushima and Ichiro TaKano \\ Tokyo Metropolitan Institute of Public Health: \\ 3-24-1 Hyakunin-cho, Shinjuku-ku, Tokyo 169-0073, Japan; \\ ${ }^{*}$ Corresponding author
}

\begin{abstract}
A method for rapid analysis of multi-class residual veterinary drugs in livestock products was developed and validated in accordance with the Japanese guideline for pesticides. Using LC-MS/ MS, 43 multi-class veterinary drugs, including sulfonamides, quinolones, coccidiostats and antiparasites, could be analyzed simultaneously in only 18 minutes. The extraction process was developed by modifying the QuEChERS approach to provide faster and less expensive extraction. The samples were extracted by using two kinds of solvent, acetonitrile and acetonitrile including 1 vol\% formic acid, and salted out with magnesium sulfate, trisodium citrate and sodium chloride. Using these two extractants, 40 out of 43 drugs satisfied the guideline criteria in bovine muscle and swine muscle, 39 drugs were found in chicken muscle, and 37 drugs were found in eggs. The limit of quantification was less than the MRL for all analytes.
\end{abstract}

Key words: veterinary drug; livestock product; LC-MS/MS; multi-class analysis; QuEChERS approach

\begin{abstract}
Veterinary drugs are often used on farms to treat and prevent diseases. However over-dosing and noncompliance with the discontinuation period could cause the accumulation of drugs in animal tissues; therefore there is a risk of human consumption of drug-contaminated livestock products that could lead to emergence of drug-resistant bacteria, allergic reaction and toxicity ${ }^{1)}$.

Veterinary drugs are divided into many classes, including synthetic antibacterials, antibiotics, antiparasites, hormones and pesticides. Each class covers a wide variety of chemical and physical properties from hydrophilic to lipophilic, and acidic to basic; therefore it is difficult to simultaneously analyze many drugs even in the same class. Although many reports have described simultaneous analysis of multiple veterinary drugs in livestock products ${ }^{2-7)}$, they do not cover the most commonly used drugs on farms, i.e., the drugs most frequently detected and reported at testing institutions such as quarantine stations. Furthermore, the sample solutions in the previous analytical methods require considerable time to prepare and are expensive, because they require a range of solvents, reagents and experi-
\end{abstract}

\footnotetext{
* Takayuki_1_Nakajima@member.metro.tokyo.jp

*1 Guidelines for the Validation of Analytical Method for Residual Agricultural Chemicals in Food (November 15, 2007, Notification No. 1115001). The Ministry of Health, Labour and Welfare of Japan.
}

mental instruments.

In recent years, the QuEChERS (Quick, Easy, Cheap, Effective, Rugged and Safe) approach has attracted great interest $^{8-15)}$. However, there are few reports on residual veterinary drugs in livestock samples ${ }^{16-21)}$. In addition, the reported methods could not analyze multiclass drugs, such as polyethers and macrolides that are frequently used and detected in livestock.

The aim of this study was to develop a rapid and easy analytical method for residual veterinary drugs in livestock products by modifying the QuEChERS approach, and to validate the method in accordance with the Japanese guidelines for pesticides ${ }^{* 1}$.

\section{Materials and Methods}

\section{Samples}

Samples (bovine muscle, swine muscle, chicken muscle and eggs) were purchased from local supermarkets in Tokyo and were confirmed to be free of the targeted drugs. The tissues were minced with an electric household food processor and stored at $-20^{\circ} \mathrm{C}$.

\section{Chemicals and reagents}

Powdered standards for testing residual veterinary drugs were purchased from Dr Ehrenstorfer (Augsburg, Germany), Hayashi Pure Chemical Industries (Osaka, Japan), Kanto Chemical Company (Tokyo, Japan), LKT Laboratories (St. Paul, U.S.A.), Sigma-Aldrich (St. 
Louis, U.S.A.) and Wako Pure Chemical Industries, Ltd. (Osaka, Japan). Stock standard solutions of individual compounds were prepared as described below. Portions of approximately $5 \mathrm{mg}$ of fenbendazole, flubendazole, nicarbazin, oxfendazole and oxfendazole sulfone powder were weighed and dissolved in $5 \mathrm{~mL}$ of dimethylformamide (HPLC grade), then diluted with methanol (HPLC grade) to $50 \mathrm{~mL}$. Decoquinate was dissolved in $5 \mathrm{~mL}$ chloroform (analytical grade) and then diluted with methanol. Oxolinic acid, pyrimethamine and sulfadimidine were dissolved in $50 \mathrm{~mL}$ of acetonitrile (HPLC grade), and other compounds were dissolved in $50 \mathrm{~mL}$ of methanol. These solutions were stored at $-20^{\circ} \mathrm{C}$. A working standard solution was prepared by mixing $500 \mu \mathrm{L}$ of all stock standard solutions and diluting the mixture with acetonitrile to $50 \mathrm{~mL}$. When stored at $4^{\circ} \mathrm{C}$, this solution was stable for three months.

Acetonitrile, distilled water (HPLC grade), methanol, formic acid (LC/MS grade), ammonium acetate (LC/MS grade), magnesium sulfate (analytical grade) and sodium chloride (analytical grade) were purchased from Wako Pure Chemical Industries. Trisodium citrate dehydrate (analytical grade) and dimethylformamide were purchased from Kanto Chemical Company.

\section{$L C-M S / M S$ conditions}

LC-MS/MS conditions are shown in Table 1.

Optimization was performed by direct infusion of $1 \mu \mathrm{g} /$ $\mathrm{mL}$ of each standard solution, and the optimized conditions for selective reaction monitoring (SRM) transitions are presented in Table 2.

\section{Sample preparation}

A $5 \mathrm{~g}$ aliquot of each minced sample was weighed into a $50 \mathrm{~mL}$ polypropylene centrifuge tube and then $5 \mathrm{~mL}$ of distilled water and $15 \mathrm{~mL}$ of $1 \mathrm{vol} \%$ formic acid in acetonitrile $(\operatorname{method} \mathrm{A})$ or acetonitrile $(\operatorname{method} \mathrm{B})$ were added. The mixture was homogenized thoroughly using an Ultra-Turrax T25 (IKA), then $4 \mathrm{~g}$ of magnesium sulfate, $1.5 \mathrm{~g}$ of trisodium citrate dehydrate and $2 \mathrm{~g}$ of sodium chloride were added, and the tube was vortexed immediately for $1 \mathrm{~min}$. The mixture was centrifuged at $1,800 \times g$ for 10 min with a multipurpose refrigerated centrifuge
AX-320 (Tomy), and the supernatant was transferred into a $20 \mathrm{~mL}$ volumetric flask. After dilution with $1 \mathrm{vol} \%$ formic acid in acetonitrile or acetonitrile to volume, a portion of the solution was transferred to a $1.5 \mathrm{~mL}$ microtube and centrifuged at $16,000 \times g$ for 10 min with a microcentrifuge 5415R (Eppendorf). Three $\mu \mathrm{L}$ of supernatant was injected into the LC-MS/MS.

\section{Quantification}

Matrix-matched calibration curves were prepared. Blank samples were prepared as above, and then matrix-matched standard solutions were prepared by mixing $900 \mu \mathrm{L}$ of blank solution and $100 \mu \mathrm{L}$ of an appropriately diluted working solution. The final concentrations of matrix-matched standard solutions were $1,2.5,10,25$ and $50 \mu \mathrm{g} / \mathrm{L}$.

\section{Method validation}

The method was validated according to the "Guidelines for the Validation of Analytical Methods for Residual Agricultural Chemicals in Food" by the Ministry of Health, Labour and Welfare, Japan. Accuracy, repeatability and within-laboratory reproducibility were determined by means of fortified recovery tests at using two different concentrations, 10 and $100 \mu \mathrm{g} / \mathrm{kg}$, performed with two samples per day on five different days. The results were evaluated by one-way analysis of variance. Limits of quantification (LOQ) were calculated by analyzing blank samples fortified at 0.25, 0.5, 1, 2 and $2.5 \mu \mathrm{g} / \mathrm{kg}$ and they were determined as the lowest concentration of an analyte for which the signal-to-noise ratio was over 10 . Selectivity was determined by analyzing blank samples.

\section{Results and Discussion}

\section{$L C-M S / M S$ conditions}

With reference to previous reports ${ }^{1)-4), 22)}$, a mobile phase consisting of $0.1 \mathrm{vol} \%$ formic acid in $10 \mathrm{mmol} / \mathrm{L}$ ammonium acetate and acetonitrile was chosen. The ammonium-adduct ions were most sensitively detected for ivermectin and doramectin, and the deprotonated ions for diclazuril, florfenicol, nicarbazin, triclabendazole sulfone and triclabendazole sulfoxide, while for the oth-

Table 1. LC-MS/MS conditions

$\begin{array}{ll}\text { HPLC system } & \text { Prominence series (Shimadzu Corporation) } \\ \text { HPLC column } & \text { Syncronis aQ }(2.1 \mathrm{~mm} \mathrm{i.d.} \times 100 \mathrm{~mm}, 5 \mu \mathrm{m} \text {, Thermo Scientific) } \\ \text { Column temperature } & 40^{\circ} \mathrm{C} \\ \text { Flow rate } & 0.3 \mathrm{~mL} / \mathrm{min} \\ \text { Mobile phase } & (\mathrm{A}) 0.1 \text { vol\% formic acid in } 10 \mathrm{mmol} / \mathrm{L} \text { ammonium acetate } \\ & (\mathrm{B}) \text { acetonitrile } \\ \text { Gradient } & (\mathrm{A} \%: \mathrm{B} \%)=0 \mathrm{~min}(95: 5) \rightarrow 7 \mathrm{~min}(5: 95) \rightarrow 12 \mathrm{~min}(5: 95) \rightarrow 12.1 \mathrm{~min}(95: 5) \rightarrow 17 \mathrm{~min}(95: 5) \\ \text { Injection volume } & 3 \mu \mathrm{L} \\ \text { MS system } & \text { TSQ Quantum Access MAX (Thermo Scientific) } \\ \text { Ionization } & \text { ESI positve, negative } \\ \text { Analysis mode } & \text { Selective reaction monitoring }(\mathrm{SRM}) \\ \text { Ion-spray voltage } & (\text { positive }) 3,000 \mathrm{~V}(\text { negative }) 2,500 \mathrm{~V} \\ \text { Vaporizer temperature } & 460^{\circ} \mathrm{C} \\ \text { Capillary temperature } & 220^{\circ} \mathrm{C}\end{array}$


Table 2. MS/MS parameters for each analyte

\begin{tabular}{|c|c|c|c|c|c|c|}
\hline \multirow[b]{2}{*}{ Analyte } & \multirow[b]{2}{*}{ R.T. (min) } & \multicolumn{5}{|c|}{ SRM transition } \\
\hline & & ESI & $\begin{array}{l}\text { Precursor ion } \\
\quad(m / z)\end{array}$ & $\begin{array}{l}\text { Product ion } \\
\quad(\mathrm{m} / \mathrm{z})\end{array}$ & $\begin{array}{l}\text { Collision } \\
\text { energy }(\mathrm{eV})\end{array}$ & Tube lens (V) \\
\hline Clopidol & 2.58 & + & 192 & 101 & 24 & 122 \\
\hline 5-Hydroxythiabendazole & 2.67 & + & 218 & 108 & 37 & 105 \\
\hline Levamisole & 2.80 & + & 205 & 91 & 35 & 102 \\
\hline $\begin{array}{l}\text { 5-Propylsulfonyl- } 1 H \text { - } \\
\text { benzimidazole-2-amine }\end{array}$ & 2.83 & + & 240 & 133 & 30 & 82 \\
\hline Sulfathiazole & 2.83 & + & 256 & 92 & 26 & 109 \\
\hline Trimethoprim & 3.01 & + & 291 & 123 & 26 & 94 \\
\hline Sulfamerazine & 3.07 & + & 265 & 92 & 29 & 93 \\
\hline Ciprofloxacin & 3.11 & + & 332 & 314 & 20 & 119 \\
\hline Ormethoprim & 3.17 & + & 275 & 123 & 21 & 107 \\
\hline Enrofloxacin & 3.31 & + & 360 & 316 & 18 & 116 \\
\hline Thiabendazole & 3.33 & + & 202 & 65 & 40 & 106 \\
\hline Sulfadimidine & 3.33 & + & 279 & 186 & 16 & 93 \\
\hline Sarafloxacin & 3.60 & + & 386 & 368 & 26 & 138 \\
\hline Sulfamonomethoxine & 3.60 & + & 281 & 108 & 25 & 82 \\
\hline Sulfamethoxazole & 3.87 & + & 254 & 92 & 25 & 96 \\
\hline Florfenicol & 3.91 & - & 356 & 336 & 13 & -87 \\
\hline Oxfendazole & 3.91 & + & 316 & 159 & 31 & 120 \\
\hline Pyrimethamine & 4.03 & + & 249 & 177 & 28 & 114 \\
\hline Oxolinic acid & 4.05 & + & 262 & 244 & 19 & 106 \\
\hline Sulfaquinoxaline & 4.27 & + & 301 & 108 & 26 & 95 \\
\hline Sulfadimethoxine & 4.27 & + & 311 & 156 & 20 & 96 \\
\hline Oxfendazole sulfone & 4.43 & + & 332 & 300 & 22 & 118 \\
\hline Nalidixic acid & 4.68 & + & 233 & 215 & 13 & 97 \\
\hline Flubendazole & 4.84 & + & 314 & 123 & 34 & 123 \\
\hline Fenbendazole & 5.51 & + & 300 & 268 & 19 & 103 \\
\hline Nicarbazin & 5.94 & - & 301 & 137 & 16 & -65 \\
\hline Triclabendazole sulfoxide & 5.90 & - & 373 & 181 & 46 & -78 \\
\hline Nequinate & 6.18 & + & 366 & 145 & 47 & 100 \\
\hline Buquinolate & 6.22 & + & 362 & 204 & 35 & 95 \\
\hline Diclazuril & 6.23 & - & 405 & 334 & 22 & -70 \\
\hline Triclabendazole sulfone & 6.25 & - & 391 & 312 & 29 & -102 \\
\hline Triclabendazole & 6.65 & + & 359 & 198 & 25 & 124 \\
\hline Decoquinate & 7.20 & + & 418 & 204 & 41 & 107 \\
\hline Eprinomectin & 7.20 & + & 915 & 112 & 55 & 113 \\
\hline Semduramicin & 7.95 & + & 891 & 630 & 22 & 126 \\
\hline Doramectin & 8.10 & + & 917 & 331 & 24 & 138 \\
\hline Lasalocid & 8.25 & + & 609 & 237 & 23 & 112 \\
\hline Moxidectin & 8.39 & + & 641 & 499 & 12 & 113 \\
\hline Ivermectin & 8.49 & + & 893 & 570 & 13 & 113 \\
\hline Maduramicin & 8.73 & + & 935 & 630 & 21 & 132 \\
\hline Salinomycin & 8.79 & + & 769 & 734 & 17 & 94 \\
\hline Monensin & 8.84 & + & 689 & 461 & 23 & 119 \\
\hline Narasin & 9.16 & + & 783 & 748 & 17 & 114 \\
\hline
\end{tabular}

ers, protonated forms were most sensitively detected.

Next, HPLC columns were examined, because analysis of multi-class drugs from hydrophilic compounds such as like sulfonamides to hydrophobic compounds such as polyethers is difficult, particularly from the viewpoints of peak shapes and good separation. L-column2 ODS (Chemicals Evaluation Research Institute), Atlantis T3 (Watres), Accucore RP-MS (Thermo Scientific) and Syncronis aQ (Thermo Scientific) were compared. Though they all basically have ODS chains, except for Accucore RP-MS (which has optimized alkyl chain length and slightly weaker retention than ODS),
Atlantis T3 and Syncronis aQ were the most suitable for highly polar compounds because they are more retentive than other conventional ODS columns.

Using L-column2 ODS, the peak shapes of sulfadimidine and sulfamerazine were poor, showing splitting. Although Atlantis T3 produced good peak shapes for sulfonamides, polyethers such as lasalocid and narasin could not be eluted from the column. In contrast, Accucore RP-MS produced good chromatograms of macrolides and polyethers, but hydrophilic analytes were not retained in the column. On the other hand, Syncronis aQ showed good retention of polar analytes, and it could 
also release lipophilic analytes, as well as providing good separation and peak shapes. Therefore, we adopted Syncronis aQ.

\section{Extraction process}

The extraction process was developed using chicken muscle that was confirmed not to contain the target analytes. All studies below were performed by fortifying $50 \mu \mathrm{L}$ of $1 \mu \mathrm{g} / \mathrm{mL}$ working standard mixture into this sample, and acetonitrile was used as the extraction sol- vent. With reference to the previous report ${ }^{16)}$, the method was modified as described below, and we also changed the clean-up step from filtration to high-speed centrifugation.

First, buffering effects were compared between sodium acetate buffer and sodium citrate buffer. Using acetate buffer, less than $40 \%$ of the target analytes were recovered; especially, quinolones, polyethers and macrolides showed very low recoveries. On the other hand, more than $60 \%$ of all analytes, except fluoroquinolones,

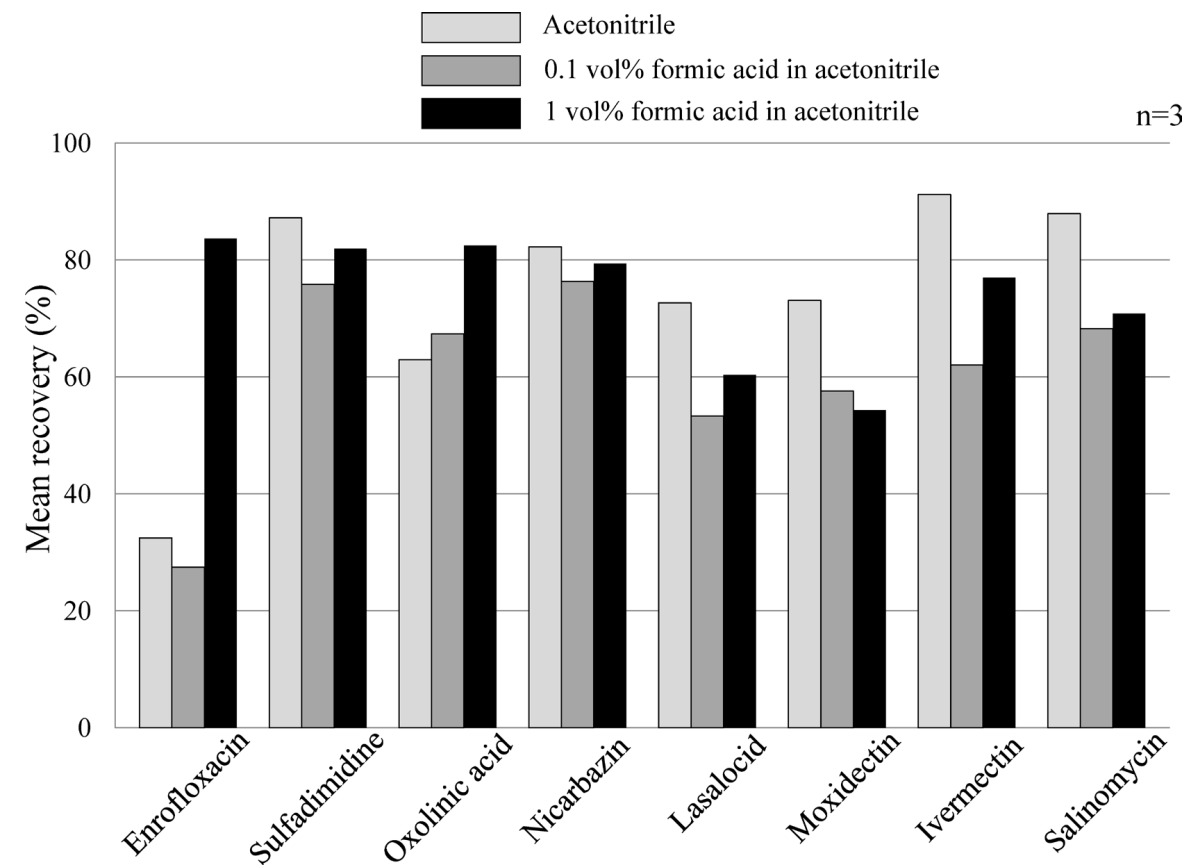

Fig. 1. Effect of the concentration of formic acid in the extraction solvent on recovery of $10 \mu \mathrm{g} / \mathrm{kg}$ standard apiked into chicken muscle

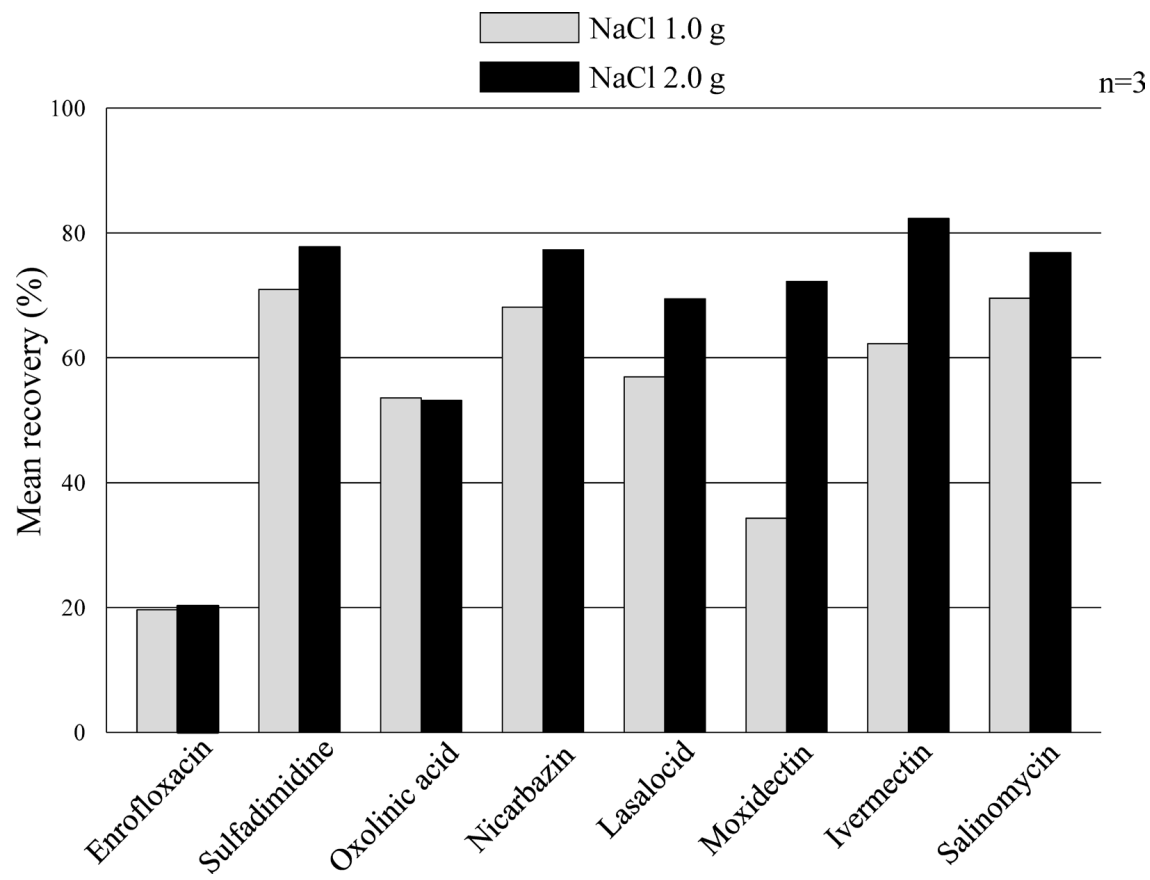

Fig. 2. Effect of different amounts of sodium chloride for salting out on recovery of $10 \mu \mathrm{g} / \mathrm{kg}$ standard spiked into chicken muscle 
Table 3. Validation results of method A

\begin{tabular}{|c|c|c|c|c|c|c|c|c|}
\hline \multirow[b]{2}{*}{ Analyte } & & Bovir & muscle & & & Swin & muscle & \\
\hline & $\begin{array}{c}\text { Fortified } \\
(\mu \mathrm{g} / \mathrm{g})\end{array}$ & $\underset{(\%)}{\text { Accuracy }}$ & $\begin{array}{c}\text { Repeat- } \\
\text { ability (\%) }\end{array}$ & $\begin{array}{l}\text { Within } \\
\text { laboratory } \\
\text { reproduci- } \\
\text { bility (\%) }\end{array}$ & $\begin{array}{c}\text { Fortified } \\
(\mu \mathrm{g} / \mathrm{g})\end{array}$ & $\underset{(\%)}{\text { Accuracy }}$ & $\begin{array}{l}\text { Repeat- } \\
\text { ability (\%) }\end{array}$ & $\begin{array}{l}\text { Within } \\
\text { laboratory } \\
\text { reproduci- } \\
\text { bility (\%) }\end{array}$ \\
\hline Clopidol & 0.01 & 59.9 & 6.1 & 7.5 & 0.01 & 57.4 & 12.2 & 17.5 \\
\hline & 0.1 & 64.4 & 9.2 & 21.8 & 0.1 & 64.4 & 9.7 & 9.1 \\
\hline 5-Hydroxythiabendazole & 0.01 & 43.8 & 18.0 & 22.9 & 0.01 & 51.7 & 31.3 & 37.8 \\
\hline & 0.1 & 51.6 & 16.5 & 17.0 & 0.1 & 61.5 & 9.9 & 10.8 \\
\hline Levamisole & 0.01 & 79.1 & 6.5 & 7.0 & 0.01 & 74.1 & 4.0 & 13.4 \\
\hline & 0.1 & 76.7 & 4.5 & 9.8 & 0.1 & 74.4 & 3.6 & 5.8 \\
\hline 5-Propylsulfonyl- $1 H$ - & 0.01 & 60.0 & 6.6 & 9.5 & 0.01 & 57.6 & 11.6 & 14.8 \\
\hline benzimidazole-2-amine & 0.1 & 63.8 & 4.9 & 5.3 & 0.1 & 66.6 & 5.5 & 7.2 \\
\hline Sulfathiazole & 0.01 & 67.1 & 11.6 & 12.5 & 0.01 & 76.0 & 7.1 & 8.9 \\
\hline & 0.1 & 78.6 & 4.8 & 12.8 & 0.1 & 85.8 & 3.6 & 6.5 \\
\hline Trimethoprim & 0.01 & 70.4 & 3.6 & 4.7 & 0.01 & 68.6 & 7.2 & 8.0 \\
\hline & 0.1 & 74.9 & 5.7 & 9.1 & 0.1 & 76.3 & 3.6 & 4.1 \\
\hline Sulfamerazine & 0.01 & 74.4 & 5.6 & 11.7 & 0.01 & 77.7 & 12.4 & 14.7 \\
\hline & 0.1 & 82.3 & 2.3 & 9.6 & 0.1 & 85.3 & 3.4 & 4.9 \\
\hline Ciprofloxacin & 0.01 & 46.7 & 11.6 & 11.9 & 0.01 & 45.1 & 10.0 & 11.9 \\
\hline & 0.1 & 42.9 & 10.6 & 10.8 & 0.1 & 50.0 & 5.8 & 8.1 \\
\hline Ormethoprim & 0.01 & 71.9 & 5.5 & 5.9 & 0.01 & 71.1 & 6.3 & 7.9 \\
\hline & 0.1 & 72.7 & 4.4 & 12.0 & 0.1 & 77.7 & 3.4 & 3.9 \\
\hline Enrofloxacin & 0.01 & 73.3 & 1.6 & 2.1 & 0.01 & 65.8 & 5.7 & 5.8 \\
\hline & 0.1 & 70.9 & 3.5 & 9.5 & 0.1 & 73.0 & 3.0 & 3.7 \\
\hline Thiabendazole & 0.01 & 59.7 & 8.0 & 12.7 & 0.01 & 63.2 & 11.0 & 11.6 \\
\hline & 0.1 & 67.0 & 6.0 & 13.3 & 0.1 & 59.8 & 6.1 & 7.2 \\
\hline Sulfadimidine & 0.01 & 83.1 & 2.4 & 3.1 & 0.01 & 82.1 & 4.8 & 7.8 \\
\hline & 0.1 & 83.2 & 2.0 & 5.3 & 0.1 & 84.3 & 2.5 & 4.1 \\
\hline Sarafloxacin & 0.01 & 66.0 & 6.1 & 9.0 & 0.01 & 70.0 & 8.0 & 9.1 \\
\hline & 0.1 & 73.3 & 3.7 & 6.2 & 0.1 & 70.9 & 1.6 & 3.5 \\
\hline Sulfamonomethoxine & 0.01 & 81.6 & 2.3 & 3.5 & 0.01 & 79.9 & 4.0 & 6.7 \\
\hline & 0.1 & 79.5 & 1.8 & 9.5 & 0.1 & 87.2 & 2.0 & 3.9 \\
\hline Sulfamethoxazole & 0.01 & 80.9 & 5.5 & 5.7 & 0.01 & 85.5 & 8.6 & 11.3 \\
\hline & 0.1 & 85.1 & 3.5 & 9.3 & 0.1 & 93.6 & 1.8 & 3.8 \\
\hline Florfenicol & 0.01 & 78.0 & 9.8 & 10.2 & 0.01 & 78.1 & 5.6 & 7.0 \\
\hline & 0.1 & 87.0 & 5.4 & 7.0 & 0.1 & 85.0 & 8.3 & 8.3 \\
\hline Oxfendazole & 0.01 & 86.4 & 1.3 & 3.2 & 0.01 & 77.4 & 4.8 & 7.6 \\
\hline & 0.1 & 79.2 & 1.4 & 4.2 & 0.1 & 79.3 & 2.2 & 5.2 \\
\hline Pyrimethamine & 0.01 & 75.7 & 1.4 & 2.3 & 0.01 & 73.5 & 4.7 & 7.9 \\
\hline & 0.1 & 75.8 & 2.9 & 6.3 & 0.1 & 76.6 & 2.1 & 3.8 \\
\hline Oxolinic acid & 0.01 & 81.5 & 1.6 & 3.1 & 0.01 & 80.9 & 3.6 & 7.1 \\
\hline & 0.1 & 81.9 & 2.5 & 7.4 & 0.1 & 79.8 & 1.2 & 3.5 \\
\hline Sulfaquinoxaline & 0.01 & 80.3 & 7.4 & 8.7 & 0.01 & 92.9 & 7.0 & 8.2 \\
\hline & 0.1 & 81.3 & 2.0 & 11.0 & 0.1 & 101.7 & 2.7 & 5.1 \\
\hline Sulfadimethoxine & 0.01 & 81.7 & 4.2 & 4.2 & 0.01 & 86.2 & 4.2 & 6.4 \\
\hline & 0.1 & 86.3 & 2.5 & 4.9 & 0.1 & 93.8 & 2.6 & 4.9 \\
\hline Oxfendazole sulfone & 0.01 & 86.0 & 2.2 & 3.2 & 0.01 & 84.1 & 4.1 & 6.8 \\
\hline & 0.1 & 84.4 & 4.5 & 5.1 & 0.1 & 84.3 & 3.3 & 5.0 \\
\hline Nalidixic acid & 0.01 & 82.5 & 2.5 & 3.9 & 0.01 & 81.2 & 4.6 & 5.9 \\
\hline & 0.1 & 82.8 & 2.5 & 7.2 & 0.1 & 82.2 & 1.6 & 4.2 \\
\hline Flubendazole & 0.01 & 81.9 & 2.5 & 2.6 & 0.01 & 81.5 & 3.4 & 6.8 \\
\hline & 0.1 & 81.2 & 9.0 & 9.3 & 0.1 & 81.4 & 3.7 & 6.1 \\
\hline Fenbendazole & 0.01 & 70.4 & 3.0 & 5.6 & 0.01 & 81.4 & 4.0 & 9.0 \\
\hline & 0.1 & 78.3 & 11.5 & 11.9 & 0.1 & 75.8 & 4.1 & 8.4 \\
\hline Nicarbazin & 0.01 & 75.7 & 4.8 & 7.2 & 0.01 & 80.9 & 5.4 & 5.5 \\
\hline & 0.1 & 85.4 & 2.6 & 2.8 & 0.1 & 70.4 & 2.2 & 4.0 \\
\hline Triclabendazole sulfoxide & 0.01 & 72.3 & 5.0 & 8.2 & 0.01 & 71.6 & 7.0 & 11.3 \\
\hline & 0.1 & 80.4 & 2.6 & 5.1 & 0.1 & 71.0 & 5.3 & 5.9 \\
\hline Nequinate & 0.01 & 70.4 & 3.7 & 6.3 & 0.01 & 74.9 & 3.0 & 5.4 \\
\hline & 0.1 & 72.0 & 7.5 & 9.4 & 0.1 & 74.1 & 2.2 & 4.6 \\
\hline Buquinolate & 0.01 & 77.8 & 3.2 & 3.9 & 0.01 & 78.2 & 3.6 & 6.8 \\
\hline & 0.1 & 75.0 & 4.7 & 6.6 & 0.1 & 77.1 & 2.4 & 5.6 \\
\hline Diclazuril & 0.01 & 84.6 & 7.6 & 10.0 & 0.01 & 82.5 & 12.1 & 14.1 \\
\hline & 0.1 & 88.5 & 4.4 & 6.4 & 0.1 & 77.7 & 3.3 & 4.3 \\
\hline Triclabendazole sulfone & 0.01 & 80.6 & 5.9 & 7.0 & 0.01 & 84.5 & 4.6 & 4.7 \\
\hline & 0.1 & 91.5 & 5.3 & 6.2 & 0.1 & 79.3 & 2.5 & 3.3 \\
\hline Triclabendazole & 0.01 & 62.9 & 6.2 & 10.4 & 0.01 & 79.5 & 5.2 & 8.5 \\
\hline & 0.1 & 69.6 & 10.1 & 15.4 & 0.1 & 64.7 & 13.4 & 13.6 \\
\hline Decoquinate & 0.01 & 95.9 & 4.8 & 7.0 & 0.01 & 87.5 & 7.6 & 10.6 \\
\hline & 0.1 & 87.8 & 5.0 & 9.2 & 0.1 & 108.2 & 4.7 & 12.9 \\
\hline Eprinomectin & 0.01 & 66.1 & 13.8 & 19.3 & 0.01 & 75.9 & 25.2 & 65.7 \\
\hline & 0.1 & 84.0 & 12.2 & 15.0 & 0.1 & 62.4 & 18.4 & 24.6 \\
\hline Semduramicin & 0.01 & 58.0 & 24.5 & 34.5 & 0.01 & 83.6 & 5.6 & 8.7 \\
\hline & 0.1 & 94.8 & 10.9 & 11.4 & 0.1 & 47.1 & 24.8 & 36.6 \\
\hline Doramectin & 0.01 & 45.1 & 42.2 & 50.6 & 0.01 & 71.2 & 22.5 & 27.2 \\
\hline & 0.1 & 72.6 & 6.8 & 18.0 & 0.1 & 43.2 & 22.0 & 30.1 \\
\hline Lasalocid & 0.01 & 58.2 & 13.7 & 14.5 & 0.01 & 73.0 & 6.5 & 11.5 \\
\hline & 0.1 & 70.0 & 5.9 & 8.7 & 0.1 & 55.2 & 7.0 & 20.7 \\
\hline Moxidectin & 0.01 & 47.3 & 17.2 & 17.8 & 0.01 & 80.1 & 18.1 & 18.4 \\
\hline & 0.1 & 77.1 & 6.5 & 14.1 & 0.1 & 41.4 & 18.1 & 24.7 \\
\hline Ivermectin & 0.01 & 63.6 & 8.3 & 10.7 & 0.01 & 82.2 & 9.9 & 11.2 \\
\hline & 0.1 & 80.6 & 8.9 & 11.7 & 0.1 & 55.2 & 8.3 & 19.3 \\
\hline Maduramicin & 0.01 & 91.3 & 5.1 & 6.4 & 0.01 & 81.2 & 5.7 & 11.1 \\
\hline & 0.1 & 89.9 & 5.2 & 9.3 & 0.1 & 81.8 & 6.4 & 8.1 \\
\hline Salinomycin & 0.01 & 66.0 & 5.2 & 8.6 & 0.01 & 79.3 & 7.2 & 12.0 \\
\hline & 0.1 & 93.2 & 4.2 & 8.4 & 0.1 & 69.8 & 5.6 & 8.8 \\
\hline Monensin & 0.01 & 71.8 & 7.6 & 7.8 & 0.01 & 81.5 & 9.5 & 11.9 \\
\hline & 0.1 & 88.2 & 7.6 & 7.6 & 0.1 & 67.1 & 9.6 & 12.9 \\
\hline Narasin & 0.01 & 78.4 & 11.9 & 13.3 & 0.01 & 82.3 & 5.8 & 6.9 \\
\hline & 0.1 & 93.2 & 3.3 & 6.5 & 0.1 & 75.7 & 2.9 & 7.2 \\
\hline
\end{tabular}


Table 3. Continued

\begin{tabular}{|c|c|c|c|c|c|c|c|c|}
\hline \multirow[b]{2}{*}{ Analyte } & & Chick & muscle & & & & $g$ & \\
\hline & $\begin{array}{c}\text { Fortified } \\
(\mu \mathrm{g} / \mathrm{g})\end{array}$ & $\begin{array}{c}\text { Accuracy } \\
(\%)\end{array}$ & $\begin{array}{l}\text { Repeat- } \\
\text { ability (\%) }\end{array}$ & $\begin{array}{l}\text { Within } \\
\text { laboratory } \\
\text { reproduci- } \\
\text { bility }(\%)\end{array}$ & $\begin{array}{c}\text { Fortified } \\
(\mu \mathrm{g} / \mathrm{g})\end{array}$ & $\begin{array}{c}\text { Accuracy } \\
(\%)\end{array}$ & $\begin{array}{l}\text { Repeat- } \\
\text { ability (\%) }\end{array}$ & $\begin{array}{l}\text { Within } \\
\text { laboratory } \\
\text { reproduci- } \\
\text { bility (\%) }\end{array}$ \\
\hline Clopidol & 0.01 & 63.6 & 7.1 & 7.7 & 0.01 & 76.6 & 11.9 & 21.5 \\
\hline & 0.1 & 71.6 & 7.9 & 22.0 & 0.1 & 89.2 & 9.8 & 27.4 \\
\hline 5-Hydroxythiabendazole & 0.01 & 52.7 & 15.9 & 16.6 & 0.01 & 71.1 & 17.7 & 20.1 \\
\hline & 0.1 & 43.5 & 17.3 & 21.6 & 0.1 & 80.4 & 12.8 & 13.2 \\
\hline Levamisole & 0.01 & 76.3 & 8.2 & 9.1 & 0.01 & 78.3 & 8.7 & 12.8 \\
\hline & 0.1 & 81.4 & 3.9 & 6.8 & 0.1 & 76.4 & 6.1 & 8.2 \\
\hline 5-Propylsulfonyl-1H- & 0.01 & 54.2 & 10.8 & 14.0 & 0.01 & 67.2 & 10.1 & 10.2 \\
\hline benzimidazole-2-amine & 0.1 & 60.0 & 3.0 & 6.8 & 0.1 & 63.9 & 7.3 & 8.0 \\
\hline Sulfathiazole & 0.01 & 81.2 & 11.4 & 11.6 & 0.01 & 110.8 & 5.2 & 19.9 \\
\hline & 0.1 & 87.3 & 5.5 & 8.3 & 0.1 & 127.1 & 3.8 & 16.8 \\
\hline Trimethoprim & 0.01 & 68.7 & 3.5 & 5.9 & 0.01 & 80.3 & 3.5 & 8.1 \\
\hline & 0.1 & 74.8 & 3.4 & 5.7 & 0.1 & 85.2 & 3.8 & 6.2 \\
\hline Sulfamerazine & 0.01 & 79.7 & 11.9 & 13.4 & 0.01 & 90.3 & 6.7 & 9.8 \\
\hline & 0.1 & 87.7 & 3.8 & 5.4 & 0.1 & 93.1 & 3.5 & 7.6 \\
\hline Ciprofloxacin & 0.01 & 44.9 & 4.0 & 7.1 & 0.01 & 55.3 & 14.6 & 17.0 \\
\hline & 0.1 & 35.2 & 8.5 & 9.2 & 0.1 & 44.1 & 5.0 & 6.4 \\
\hline Ormethoprim & 0.01 & 70.3 & 5.3 & 6.7 & 0.01 & 87.3 & 3.3 & 10.0 \\
\hline & 0.1 & 77.7 & 4.7 & 5.4 & 0.1 & 94.9 & 2.3 & 7.5 \\
\hline Enrofloxacin & 0.01 & 70.4 & 4.3 & 7.8 & 0.01 & 86.7 & 3.5 & 4.8 \\
\hline & 0.1 & 71.3 & 3.4 & 6.0 & 0.1 & 84.4 & 4.9 & 7.4 \\
\hline Thiabendazole & 0.01 & 70.2 & 5.4 & 7.3 & 0.01 & 59.3 & 16.2 & 17.2 \\
\hline & 0.1 & 66.5 & 7.6 & 10.6 & 0.1 & 66.5 & 5.1 & 8.9 \\
\hline Sulfadimidine & 0.01 & 83.4 & 2.8 & 4.5 & 0.01 & 93.3 & 2.5 & 4.2 \\
\hline & 0.1 & 90.2 & 1.9 & 2.8 & 0.1 & 88.8 & 1.8 & 4.3 \\
\hline Sarafloxacin & 0.01 & 68.1 & 7.5 & 7.8 & 0.01 & 110.8 & 8.2 & 10.7 \\
\hline & 0.1 & 68.3 & 4.5 & 7.2 & 0.1 & 92.7 & 4.8 & 7.9 \\
\hline Sulfamonomethoxine & 0.01 & 83.1 & 3.8 & 4.6 & 0.01 & 114.4 & 3.6 & 13.5 \\
\hline & 0.1 & 88.3 & 2.3 & 3.2 & 0.1 & 100.4 & 2.6 & 12.3 \\
\hline Sulfamethoxazole & 0.01 & 80.9 & 4.1 & 9.2 & 0.01 & 108.0 & 7.0 & 13.1 \\
\hline & 0.1 & 93.2 & 3.1 & 3.2 & 0.1 & 108.0 & 2.8 & 11.2 \\
\hline Florfenicol & 0.01 & 74.1 & 7.2 & 8.6 & 0.01 & 153.9 & 10.4 & 29.0 \\
\hline & 0.1 & 84.9 & 2.7 & 11.6 & 0.1 & 128.9 & 4.0 & 18.2 \\
\hline Oxfendazole & 0.01 & 84.4 & 11.3 & 11.4 & 0.01 & 103.9 & 4.3 & 11.8 \\
\hline & 0.1 & 89.8 & 2.3 & 2.7 & 0.1 & 95.5 & 2.2 & 8.2 \\
\hline Pyrimethamine & 0.01 & 73.2 & 5.5 & 5.7 & 0.01 & 82.5 & 2.4 & 4.2 \\
\hline & 0.1 & 80.1 & 3.3 & 3.6 & 0.1 & 75.3 & 2.6 & 4.9 \\
\hline Oxolinic acid & 0.01 & 83.4 & 1.6 & 2.2 & 0.01 & 91.2 & 2.5 & 3.7 \\
\hline & 0.1 & 87.5 & 2.6 & 3.2 & 0.1 & 82.8 & 1.8 & 4.0 \\
\hline Sulfaquinoxaline & 0.01 & 94.8 & 3.5 & 5.4 & 0.01 & 135.4 & 5.7 & 22.1 \\
\hline & 0.1 & 109.0 & 3.0 & 3.0 & 0.1 & 187.8 & 2.7 & 22.6 \\
\hline Sulfadimethoxine & 0.01 & 90.9 & 1.6 & 2.1 & 0.01 & 99.5 & 2.6 & 8.8 \\
\hline & 0.1 & 103.3 & 2.6 & 2.8 & 0.1 & 132.0 & 1.4 & 8.6 \\
\hline Oxfendazole sulfone & 0.01 & 92.7 & 2.2 & 4.4 & 0.01 & 104.8 & 3.8 & 9.2 \\
\hline & 0.1 & 95.6 & 2.7 & 3.0 & 0.1 & 235.2 & 2.9 & 7.2 \\
\hline Nalidixic acid & 0.01 & 85.6 & 2.2 & 4.3 & 0.01 & 109.2 & 1.6 & 10.0 \\
\hline & 0.1 & 90.7 & 2.1 & 4.4 & 0.1 & 184.3 & 2.8 & 12.5 \\
\hline Flubendazole & 0.01 & 92.5 & 4.8 & 4.8 & 0.01 & 121.5 & 1.8 & 14.6 \\
\hline & 0.1 & 96.6 & 2.3 & 4.9 & 0.1 & 221.0 & 1.9 & 15.6 \\
\hline Fenbendazole & 0.01 & 91.7 & 2.2 & 3.0 & 0.01 & 99.4 & 2.1 & 8.9 \\
\hline & 0.1 & 95.5 & 2.9 & 4.2 & 0.1 & 91.8 & 1.7 & 8.3 \\
\hline Nicarbazin & 0.01 & 67.6 & 4.6 & 7.5 & 0.01 & 89.4 & 4.0 & 8.6 \\
\hline & 0.1 & 81.1 & 3.3 & 4.7 & 0.1 & 86.3 & 2.6 & 5.8 \\
\hline Triclabendazole sulfoxide & 0.01 & 67.2 & 17.0 & 17.5 & 0.01 & 85.8 & 7.2 & 10.3 \\
\hline & 0.1 & 76.5 & 7.6 & 7.9 & 0.1 & 87.9 & 4.0 & 5.1 \\
\hline Nequinate & 0.01 & 77.1 & 4.7 & 6.7 & 0.01 & 105.8 & 3.4 & 12.0 \\
\hline & 0.1 & 81.5 & 1.4 & 4.8 & 0.1 & 97.7 & 3.1 & 9.5 \\
\hline Buquinolate & 0.01 & 79.2 & 2.7 & 8.5 & 0.01 & 113.0 & 3.1 & 11.8 \\
\hline & 0.1 & 83.9 & 2.3 & 5.0 & 0.1 & 103.9 & 2.3 & 8.9 \\
\hline Diclazuril & 0.01 & 72.0 & 7.5 & 7.5 & 0.01 & 124.7 & 6.8 & 23.7 \\
\hline & 0.1 & 77.9 & 3.8 & 7.5 & 0.1 & 119.0 & 5.6 & 16.4 \\
\hline Triclabendazole sulfone & 0.01 & 72.5 & 5.3 & 10.2 & 0.01 & 118.9 & 1.3 & 14.3 \\
\hline & 0.1 & 79.7 & 5.2 & 9.3 & 0.1 & 112.1 & 2.2 & 7.1 \\
\hline Triclabendazole & 0.01 & 79.6 & 2.5 & 5.1 & 0.01 & 98.2 & 14.6 & 15.2 \\
\hline & 0.1 & 85.3 & 4.3 & 6.6 & 0.1 & 103.1 & 6.4 & 9.9 \\
\hline Decoquinate & 0.01 & 71.9 & 5.2 & 28.5 & 0.01 & 194.1 & 7.0 & 25.6 \\
\hline & 0.1 & 75.8 & 8.1 & 21.8 & 0.1 & 284.3 & 9.5 & 19.7 \\
\hline Eprinomectin & 0.01 & 73.2 & 36.0 & 40.1 & 0.01 & 81.9 & 16.7 & 18.0 \\
\hline & 0.1 & 83.0 & 29.2 & 33.3 & 0.1 & 135.8 & 26.9 & 30.4 \\
\hline Semduramicin & 0.01 & 65.8 & 21.3 & 24.2 & 0.01 & 85.7 & 6.9 & 21.3 \\
\hline & 0.1 & 86.4 & 16.0 & 18.4 & 0.1 & 139.3 & 9.7 & 22.7 \\
\hline Doramectin & 0.01 & 53.5 & 37.3 & 50.3 & 0.01 & 165.5 & 23.3 & 42.8 \\
\hline & 0.1 & 97.7 & 15.7 & 31.3 & 0.1 & 222.9 & 14.0 & 20.5 \\
\hline Lasalocid & 0.01 & 55.5 & 6.6 & 10.9 & 0.01 & 94.1 & 10.3 & 13.2 \\
\hline & 0.1 & 79.2 & 4.2 & 13.9 & 0.1 & 126.1 & 7.0 & 12.8 \\
\hline Moxidectin & 0.01 & 59.8 & 32.6 & 33.5 & 0.01 & 93.5 & 22.8 & 24.6 \\
\hline & 0.1 & 84.7 & 3.8 & 14.0 & 0.1 & 144.0 & 10.2 & 14.7 \\
\hline Ivermectin & 0.01 & 80.3 & 5.6 & 12.0 & 0.01 & 113.1 & 12.1 & 20.5 \\
\hline & 0.1 & 94.5 & 4.8 & 10.7 & 0.1 & 173.4 & 14.9 & 18.8 \\
\hline Maduramicin & 0.01 & 82.8 & 6.4 & 6.9 & 0.01 & 92.2 & 2.4 & 15.9 \\
\hline & 0.1 & 95.4 & 6.3 & 6.7 & 0.1 & 119.7 & 3.4 & 14.4 \\
\hline Salinomycin & 0.01 & 59.0 & 14.5 & 16.6 & 0.01 & 68.7 & 1.5 & 31.5 \\
\hline & 0.1 & 67.4 & 12.8 & 13.6 & 0.1 & 86.1 & 6.6 & 31.0 \\
\hline Monensin & 0.01 & 75.9 & 22.9 & 27.5 & 0.01 & 103.3 & 4.6 & 15.0 \\
\hline & 0.1 & 89.3 & 17.7 & 21.2 & 0.1 & 149.2 & 10.3 & 14.1 \\
\hline Narasin & 0.01 & 72.2 & 2.3 & 11.4 & 0.01 & 90.4 & 3.9 & 26.8 \\
\hline & 0.1 & 78.3 & 2.2 & 7.3 & 0.1 & 108.2 & 4.3 & 23.6 \\
\hline
\end{tabular}


Table 4. Validation results of method B

\begin{tabular}{|c|c|c|c|c|c|c|c|c|}
\hline \multirow[b]{2}{*}{ Analyte } & & Bovi & muscle & & & Swi & muscle & \\
\hline & $\begin{array}{c}\text { Fortified } \\
(\mu \mathrm{g} / \mathrm{g})\end{array}$ & $\underset{(\%)}{\text { Accuracy }}$ & $\begin{array}{c}\text { Repeat- } \\
\text { ability (\%) }\end{array}$ & $\begin{array}{l}\text { Within } \\
\text { laboratory } \\
\text { reproduci- } \\
\text { bility (\%) }\end{array}$ & $\begin{array}{c}\text { Fortified } \\
(\mu \mathrm{g} / \mathrm{g})\end{array}$ & $\underset{(\%)}{\text { Accuracy }}$ & $\begin{array}{c}\text { Repeat- } \\
\text { ability (\%) }\end{array}$ & $\begin{array}{l}\text { Within } \\
\text { laboratory } \\
\text { reproduci- } \\
\text { bility }(\%)\end{array}$ \\
\hline Clopidol & 0.01 & 87.1 & 20.9 & 23.2 & 0.01 & 83.3 & 9.5 & 10.0 \\
\hline & 0.1 & 88.5 & 10.4 & 13.1 & 0.1 & 75.5 & 8.2 & 18.5 \\
\hline 5-Hydroxythiabendazole & 0.01 & 66.5 & 35.3 & 44.2 & 0.01 & 60.8 & 27.6 & 39.3 \\
\hline & 0.1 & 90.3 & 11.4 & 13.2 & 0.1 & 69.0 & 8.9 & 16.7 \\
\hline Levamisole & 0.01 & 95.6 & 5.5 & 10.8 & 0.01 & 97.9 & 2.2 & 9.4 \\
\hline & 0.1 & 89.7 & 7.5 & 8.0 & 0.1 & 87.2 & 3.8 & 10.8 \\
\hline 5-Propylsulfonyl- $1 H$ - & 0.01 & 79.9 & 15.0 & 20.4 & 0.01 & 70.7 & 10.4 & 14.9 \\
\hline benzimidazole-2-amine & 0.1 & 85.6 & 11.0 & 11.6 & 0.1 & 70.3 & 4.2 & 7.7 \\
\hline Sulfathiazole & 0.01 & 93.1 & 7.9 & 8.1 & 0.01 & 93.7 & 8.7 & 10.5 \\
\hline & 0.1 & 94.5 & 4.6 & 7.0 & 0.1 & 93.3 & 3.0 & 14.6 \\
\hline Trimethoprim & 0.01 & 89.1 & 9.3 & 12.4 & 0.01 & 85.0 & 10.7 & 13.1 \\
\hline & 0.1 & 87.8 & 4.9 & 5.9 & 0.1 & 79.2 & 5.0 & 5.5 \\
\hline Sulfamerazine & 0.01 & 96.2 & 8.7 & 12.2 & 0.01 & 113.8 & 5.0 & 6.2 \\
\hline & 0.1 & 90.4 & 6.3 & 7.9 & 0.1 & 92.8 & 1.9 & 9.3 \\
\hline Ciprofloxacin & 0.01 & - & - & - & 0.01 & 14.6 & 4.1 & 4.2 \\
\hline & 0.1 & 4.5 & 20.9 & 24.0 & 0.1 & 2.9 & 8.3 & 13.1 \\
\hline Ormethoprim & 0.01 & 89.8 & 5.2 & 10.9 & 0.01 & 81.2 & 3.4 & 5.4 \\
\hline & 0.1 & 93.3 & 7.1 & 8.5 & 0.1 & 80.1 & 3.1 & 9.0 \\
\hline Enrofloxacin & 0.01 & 17.8 & 28.8 & 29.0 & 0.01 & 12.3 & 9.9 & 25.5 \\
\hline & 0.1 & 21.1 & 14.9 & 16.2 & 0.1 & 11.7 & 8.8 & 15.1 \\
\hline Thiabendazole & 0.01 & 84.3 & 8.5 & 9.2 & 0.01 & 98.4 & 7.4 & 11.7 \\
\hline & 0.1 & 90.9 & 5.3 & 7.7 & 0.1 & 89.3 & 2.1 & 11.5 \\
\hline Sulfadimidine & 0.01 & 93.7 & 5.9 & 6.4 & 0.01 & 101.9 & 2.9 & 4.6 \\
\hline & 0.1 & 92.0 & 3.7 & 6.6 & 0.1 & 93.3 & 2.4 & 9.5 \\
\hline Sarafloxacin & 0.01 & 20.0 & 40.1 & 43.5 & 0.01 & 14.8 & 13.4 & 20.6 \\
\hline & 0.1 & 19.7 & 15.3 & 15.4 & 0.1 & 6.7 & 14.0 & 56.6 \\
\hline Sulfamonomethoxine & 0.01 & 94.2 & 5.7 & 10.6 & 0.01 & 100.9 & 2.4 & 6.2 \\
\hline & 0.1 & 91.9 & 5.9 & 6.3 & 0.1 & 93.3 & 2.5 & 10.6 \\
\hline Sulfamethoxazole & 0.01 & 96.5 & 2.0 & 4.2 & 0.01 & 106.4 & 9.3 & 10.8 \\
\hline & 0.1 & 97.7 & 3.2 & 5.2 & 0.1 & 98.1 & 2.0 & 9.9 \\
\hline Florfenicol & 0.01 & 92.6 & 11.3 & 33.0 & 0.01 & 78.3 & 12.2 & 13.3 \\
\hline & 0.1 & 103.0 & 13.2 & 32.3 & 0.1 & 77.1 & 6.9 & 15.3 \\
\hline Oxfendazole & 0.01 & 97.1 & 18.2 & 20.8 & 0.01 & 107.9 & 5.3 & 30.0 \\
\hline & 0.1 & 91.6 & 9.7 & 10.9 & 0.1 & 86.5 & 1.5 & 6.8 \\
\hline Pyrimethamine & 0.01 & 82.4 & 7.3 & 7.4 & 0.01 & 78.4 & 15.2 & 19.5 \\
\hline & 0.1 & 86.3 & 4.3 & 4.8 & 0.1 & 84.6 & 1.8 & 7.3 \\
\hline Oxolinic acid & 0.01 & 65.8 & 20.4 & 21.8 & 0.01 & 66.4 & 2.7 & 23.0 \\
\hline & 0.1 & 59.2 & 4.8 & 5.5 & 0.1 & 51.9 & 2.8 & 4.7 \\
\hline Sulfaquinoxaline & 0.01 & 114.3 & 12.8 & 13.4 & 0.01 & 120.2 & 4.2 & 36.5 \\
\hline & 0.1 & 111.1 & 10.9 & 12.7 & 0.1 & 96.3 & 3.4 & 8.7 \\
\hline Sulfadimethoxine & 0.01 & 102.2 & 14.4 & 15.4 & 0.01 & 114.6 & 1.5 & 23.8 \\
\hline & 0.1 & 97.6 & 3.2 & 4.4 & 0.1 & 96.2 & 1.9 & 9.0 \\
\hline Oxfendazole sulfone & 0.01 & 95.8 & 15.2 & 18.5 & 0.01 & 114.4 & 10.7 & 33.1 \\
\hline & 0.1 & 89.9 & 6.7 & 7.0 & 0.1 & 87.8 & 1.7 & 4.7 \\
\hline Nalidixic acid & 0.01 & 58.3 & 17.8 & 20.2 & 0.01 & 55.0 & 4.0 & 22.8 \\
\hline & 0.1 & 52.5 & 6.0 & 7.9 & 0.1 & 43.7 & 2.3 & 5.0 \\
\hline Flubendazole & 0.01 & 99.0 & 19.1 & 20.3 & 0.01 & 115.5 & 7.9 & 34.0 \\
\hline & 0.1 & 89.0 & 7.0 & 7.6 & 0.1 & 87.1 & 1.8 & 7.6 \\
\hline Fenbendazole & 0.01 & 94.5 & 15.5 & 17.5 & 0.01 & 120.5 & 3.5 & 35.6 \\
\hline & 0.1 & 90.4 & 2.4 & 5.9 & 0.1 & 91.6 & 0.7 & 8.8 \\
\hline Nicarbazin & 0.01 & 95.3 & 7.3 & 8.5 & 0.01 & 89.1 & 5.3 & 8.8 \\
\hline & 0.1 & 88.2 & 3.0 & 5.8 & 0.1 & 78.9 & 2.4 & 7.6 \\
\hline Triclabendazole sulfoxide & 0.01 & 82.8 & 10.6 & 14.7 & 0.01 & 76.2 & 13.9 & 15.0 \\
\hline & 0.1 & 82.4 & 7.7 & 12.3 & 0.1 & 77.4 & 4.0 & 7.5 \\
\hline Nequinate & 0.01 & 108.5 & 8.0 & 9.3 & 0.01 & 99.1 & 1.7 & 7.0 \\
\hline & 0.1 & 95.7 & 8.2 & 8.3 & 0.1 & 82.0 & 2.3 & 7.0 \\
\hline Buquinolate & 0.01 & 101.8 & 6.7 & 8.3 & 0.01 & 100.3 & 2.2 & 8.7 \\
\hline & 0.1 & 91.6 & 5.2 & 7.8 & 0.1 & 84.3 & 2.1 & 6.5 \\
\hline Diclazuril & 0.01 & 94.4 & 8.5 & 12.7 & 0.01 & 83.8 & 6.4 & 13.6 \\
\hline & 0.1 & 94.0 & 9.4 & 16.9 & 0.1 & 81.3 & 1.8 & 4.5 \\
\hline Triclabendazole sulfone & 0.01 & 87.6 & 12.7 & 25.2 & 0.01 & 90.8 & 4.0 & 24.7 \\
\hline & 0.1 & 92.5 & 12.1 & 15.5 & 0.1 & 95.7 & 6.1 & 8.5 \\
\hline Triclabendazole & 0.01 & 108.2 & 21.9 & 22.6 & 0.01 & 114.6 & 4.8 & 24.0 \\
\hline & 0.1 & 97.6 & 6.7 & 12.8 & 0.1 & 89.9 & 2.5 & 11.5 \\
\hline Decoquinate & 0.01 & 126.7 & 15.7 & 18.5 & 0.01 & 89.9 & 2.2 & 19.2 \\
\hline & 0.1 & 103.7 & 8.0 & 22.8 & 0.1 & 75.5 & 3.5 & 8.1 \\
\hline Eprinomectin & 0.01 & 98.9 & 22.0 & 22.1 & 0.01 & 100.2 & 4.3 & 22.6 \\
\hline & 0.1 & 99.2 & 6.0 & 6.5 & 0.1 & 85.0 & 4.0 & 6.9 \\
\hline Semduramicin & 0.01 & 94.3 & 9.1 & 11.5 & 0.01 & 108.3 & 8.4 & 8.7 \\
\hline & 0.1 & 83.7 & 7.7 & 8.5 & 0.1 & 94.5 & 6.5 & 10.7 \\
\hline Doramectin & 0.01 & 110.0 & 17.5 & 17.6 & 0.01 & 101.4 & 10.6 & 16.1 \\
\hline & 0.1 & 87.8 & 14.7 & 17.9 & 0.1 & 93.1 & 5.5 & 7.4 \\
\hline Lasalocid & 0.01 & 81.0 & 15.5 & 16.7 & 0.01 & 81.9 & 8.0 & 13.5 \\
\hline & 0.1 & 71.9 & 8.2 & 9.1 & 0.1 & 78.9 & 3.9 & 12.0 \\
\hline Moxidectin & 0.01 & 101.1 & $16 . \overline{5}$ & 16.7 & 0.01 & 104.9 & 6.7 & 8.7 \\
\hline & 0.1 & 88.6 & 12.9 & 14.2 & 0.1 & 90.8 & 6.4 & 8.5 \\
\hline Ivermectin & 0.01 & 96.7 & 23.5 & 25.8 & 0.01 & 108.3 & 6.2 & 9.0 \\
\hline & 0.1 & 83.7 & 5.1 & 12.5 & 0.1 & 97.7 & 3.3 & 12.7 \\
\hline Maduramicin & 0.01 & 94.0 & 19.2 & 23.1 & 0.01 & 110.3 & 2.2 & 2.9 \\
\hline & 0.1 & 91.9 & 6.6 & 12.0 & 0.1 & 97.1 & 1.6 & 10.3 \\
\hline Salinomycin & 0.01 & 91.4 & 14.4 & 16.7 & 0.01 & 95.6 & 2.8 & 6.1 \\
\hline & 0.1 & 87.8 & 5.1 & 7.8 & 0.1 & 82.1 & 2.2 & 5.6 \\
\hline Monensin & 0.01 & 91.3 & 20.9 & 25.0 & 0.01 & 95.5 & 2.0 & 4.9 \\
\hline & 0.1 & 83.4 & 5.2 & 8.7 & 0.1 & 85.0 & 2.2 & 5.9 \\
\hline Narasin & 0.01 & 84.3 & 11.7 & 9.2 & 0.01 & 89.1 & 4.0 & 6.9 \\
\hline & 0.1 & 83.5 & 9.6 & 10.8 & 0.1 & 80.1 & 0.8 & 5.7 \\
\hline
\end{tabular}


Table 4. Continued

\begin{tabular}{|c|c|c|c|c|c|c|c|c|}
\hline \multirow[b]{2}{*}{ Analyte } & & Chick & n muscle & & & & gg & \\
\hline & $\begin{array}{c}\text { Fortified } \\
(\mu \mathrm{g} / \mathrm{g})\end{array}$ & $\underset{(\%)}{\text { Accuracy }}$ & $\begin{array}{l}\text { Repeat- } \\
\text { ability (\%) }\end{array}$ & $\begin{array}{l}\text { Within } \\
\text { laboratory } \\
\text { reproduc- } \\
\text { ibility(\%) }\end{array}$ & $\begin{array}{c}\text { Fortified } \\
(\mu \mathrm{g} / \mathrm{g})\end{array}$ & $\underset{(\%)}{\text { Accuracy }}$ & $\begin{array}{l}\text { Repeat- } \\
\text { ability (\%) }\end{array}$ & $\begin{array}{l}\text { Within } \\
\text { laboratory } \\
\text { reproduc- } \\
\text { ibility (\%) }\end{array}$ \\
\hline Clopidol & 0.01 & 86.1 & 8.5 & 17.2 & 0.01 & 62.9 & 8.9 & 14.1 \\
\hline & 0.1 & 91.4 & 15.0 & 17.7 & 0.1 & 70.8 & 8.6 & 10.3 \\
\hline 5-Hydroxythiabendazole & 0.01 & 83.4 & 56.1 & 63.7 & 0.01 & 57.0 & 17.9 & 39.2 \\
\hline & 0.1 & 77.3 & 14.2 & 16.1 & 0.1 & 80.5 & 12.7 & 13.9 \\
\hline Levamisole & 0.01 & 95.2 & 11.1 & 17.0 & 0.01 & 78.4 & 8.4 & 8.4 \\
\hline & 0.1 & 90.5 & 1.0 & 3.3 & 0.1 & 86.1 & 6.0 & 11.2 \\
\hline 5-Propylsulfonyl-1H- & 0.01 & 70.9 & 8.9 & 13.0 & 0.01 & 80.8 & 15.0 & 19.0 \\
\hline benzimidazole- 2 -amine & 0.1 & 77.4 & 4.2 & 9.5 & 0.1 & 87.3 & 2.1 & 14.4 \\
\hline Sulfathiazole & 0.01 & 91.8 & 10.2 & 15.7 & 0.01 & 91.2 & 15.7 & 20.3 \\
\hline & 0.1 & 90.1 & 4.9 & 7.4 & 0.1 & 87.0 & 5.8 & 18.7 \\
\hline Trimethoprim & 0.01 & 84.8 & 10.7 & 11.5 & 0.01 & 79.5 & 7.9 & 9.4 \\
\hline & 0.1 & 84.0 & 2.4 & 3.7 & 0.1 & 83.2 & 3.2 & 10.1 \\
\hline Sulfamerazine & 0.01 & 94.1 & 4.5 & 11.5 & 0.01 & 92.7 & 7.3 & 11.2 \\
\hline & 0.1 & 97.5 & 3.1 & 9.0 & 0.1 & 88.4 & 3.3 & 10.8 \\
\hline Ciprofloxacin & 0.01 & - & - & - & 0.01 & - & - & - \\
\hline & 0.1 & 3.5 & 6.8 & 36.4 & 0.1 & - & - & - \\
\hline Ormethoprim & 0.01 & 79.8 & 8.1 & 8.9 & 0.01 & 83.7 & 6.5 & 9.3 \\
\hline & 0.1 & 85.5 & 3.6 & 4.1 & 0.1 & 85.4 & 1.8 & 11.6 \\
\hline Enrofloxacin & 0.01 & 8.7 & 24.9 & 28.7 & 0.01 & - & - & - \\
\hline & 0.1 & 11.4 & 8.7 & 18.4 & 0.1 & - & - & - \\
\hline Thiabendazole & 0.01 & 91.2 & 10.0 & 12.9 & 0.01 & 79.6 & 6.0 & 9.0 \\
\hline & 0.1 & 90.6 & 4.3 & 6.5 & 0.1 & 83.0 & 4.5 & 8.3 \\
\hline Sulfadimidine & 0.01 & 97.3 & 4.1 & 7.7 & 0.01 & 90.7 & 3.0 & 4.5 \\
\hline & 0.1 & 96.5 & 2.9 & 8.0 & 0.1 & 91.2 & 2.6 & 6.4 \\
\hline Sarafloxacin & 0.01 & 8.8 & 19.9 & 22.6 & 0.01 & - & - & - \\
\hline & 0.1 & 5.8 & 10.1 & 46.6 & 0.1 & 2.0 & 15.5 & 35.3 \\
\hline Sulfamonomethoxine & 0.01 & 105.5 & 7.3 & 12.3 & 0.01 & 94.5 & 14.6 & 20.5 \\
\hline & 0.1 & 96.0 & 2.4 & 11.0 & 0.1 & 89.6 & 3.7 & 18.6 \\
\hline Sulfamethoxazole & 0.01 & 98.5 & 7.6 & 8.7 & 0.01 & 98.2 & 16.2 & 17.0 \\
\hline & 0.1 & 98.2 & 2.3 & 8.3 & 0.1 & 89.4 & 4.1 & 16.2 \\
\hline Florfenicol & 0.01 & 88.5 & 6.3 & 13.0 & 0.01 & 118.1 & 12.5 & 39.3 \\
\hline & 0.1 & 102.4 & 7.3 & 15.5 & 0.1 & 93.6 & 3.2 & 27.8 \\
\hline Oxfendazole & 0.01 & 88.6 & 7.4 & 7.9 & 0.01 & 99.4 & 5.6 & 15.8 \\
\hline & 0.1 & 90.5 & 2.0 & 3.8 & 0.1 & 96.9 & 4.2 & 12.4 \\
\hline Pyrimethamine & 0.01 & 78.5 & 11.5 & 12.9 & 0.01 & 85.3 & 1.1 & 3.5 \\
\hline & 0.1 & 86.1 & 2.3 & 7.2 & 0.1 & 85.3 & 3.1 & 6.0 \\
\hline Oxolinic acid & 0.01 & 51.5 & 10.3 & 10.5 & 0.01 & 8.6 & 15.8 & 20.9 \\
\hline & 0.1 & 50.4 & 2.7 & 8.9 & 0.1 & 11.4 & 2.6 & 18.7 \\
\hline Sulfaquinoxaline & 0.01 & 91.3 & 6.8 & 8.0 & 0.01 & 104.2 & 24.9 & 29.9 \\
\hline & 0.1 & 99.2 & 4.9 & 5.4 & 0.1 & 98.6 & 3.2 & 29.4 \\
\hline Sulfadimethoxine & 0.01 & 94.1 & 5.1 & 7.9 & 0.01 & 98.0 & 9.7 & 11.9 \\
\hline & 0.1 & 97.6 & 2.9 & 8.4 & 0.1 & 98.0 & 2.9 & 10.7 \\
\hline Oxfendazole sulfone & 0.01 & 89.4 & 12.9 & 15.1 & 0.01 & 97.3 & 3.2 & 10.5 \\
\hline & 0.1 & 89.0 & 1.5 & 6.0 & 0.1 & 97.6 & 2.8 & 10.0 \\
\hline Nalidixic acid & 0.01 & 41.7 & 5.5 & 9.4 & 0.01 & 6.3 & 2.9 & 6.7 \\
\hline & 0.1 & 40.9 & 3.4 & 7.6 & 0.1 & 9.0 & 3.2 & 16.4 \\
\hline Flubendazole & 0.01 & 88.3 & 8.6 & 8.9 & 0.01 & 100.5 & 11.7 & 19.2 \\
\hline & 0.1 & 88.2 & 2.7 & 4.8 & 0.1 & 110.5 & 5.6 & 30.2 \\
\hline Fenbendazole & 0.01 & 100.4 & 9.8 & 13.3 & 0.01 & 94.5 & 12.2 & 18.1 \\
\hline & 0.1 & 92.8 & 1.1 & 6.9 & 0.1 & 93.0 & 5.3 & 12.0 \\
\hline Nicarbazin & 0.01 & 83.3 & 7.1 & 5.5 & 0.01 & 84.9 & 3.1 & 6.1 \\
\hline & 0.1 & 84.6 & 3.0 & 4.4 & 0.1 & 81.1 & 1.8 & 5.6 \\
\hline Triclabendazole sulfoxide & 0.01 & 74.6 & 4.3 & 6.0 & 0.01 & 85.2 & 4.8 & 9.6 \\
\hline & 0.1 & 74.4 & 2.8 & 6.0 & 0.1 & 87.4 & 5.1 & 11.7 \\
\hline Nequinate & 0.01 & 84.3 & 5.1 & 6.5 & 0.01 & 87.4 & 11.5 & 13.5 \\
\hline & 0.1 & 83.1 & 2.2 & 2.2 & 0.1 & 82.1 & 2.6 & 10.0 \\
\hline Buquinolate & 0.01 & 87.9 & 5.8 & 6.7 & 0.01 & 89.8 & 10.8 & 12.4 \\
\hline & 0.1 & 86.2 & 2.6 & 3.0 & 0.1 & 83.4 & 2.4 & 10.5 \\
\hline Diclazuril & 0.01 & 91.9 & 8.8 & 9.4 & 0.01 & 95.4 & 7.0 & 13.6 \\
\hline & 0.1 & 84.3 & 2.3 & 9.0 & 0.1 & 82.3 & 1.9 & 16.2 \\
\hline Triclabendazole sulfone & 0.01 & 99.3 & 8.9 & 14.8 & 0.01 & 100.8 & 5.8 & 13.9 \\
\hline & 0.1 & 93.8 & 4.3 & 5.8 & 0.1 & 104.3 & 4.1 & 18.6 \\
\hline Triclabendazole & 0.01 & 96.7 & 13.1 & 18.0 & 0.01 & 86.4 & 9.5 & 13.3 \\
\hline & 0.1 & 89.4 & 3.2 & 7.3 & 0.1 & 79.2 & 7.5 & 12.4 \\
\hline Decoquinate & 0.01 & 81.0 & 4.0 & 11.7 & 0.01 & 90.5 & 11.5 & 15.8 \\
\hline & 0.1 & 80.2 & 1.3 & 18.4 & 0.1 & 73.9 & 3.3 & 18.8 \\
\hline Eprinomectin & 0.01 & 112.5 & 11.7 & 14.5 & 0.01 & 102.5 & 7.6 & 17.2 \\
\hline & 0.1 & 99.9 & 3.8 & 7.2 & 0.1 & 93.4 & 7.5 & 17.0 \\
\hline Semduramicin & 0.01 & 93.1 & 13.4 & 16.0 & 0.01 & 88.7 & 14.3 & 16.1 \\
\hline & 0.1 & 90.2 & 5.9 & 8.3 & 0.1 & 86.0 & 12.2 & 14.0 \\
\hline Doramectin & 0.01 & 102.2 & 16.6 & 17.2 & 0.01 & 79.4 & 7.9 & 29.2 \\
\hline & 0.1 & 105.2 & 7.5 & 8.9 & 0.1 & 79.6 & 3.9 & 19.7 \\
\hline Lasalocid & 0.01 & 83.6 & 4.4 & 24.7 & 0.01 & 70.3 & 8.3 & 9.5 \\
\hline & 0.1 & 82.8 & 12.1 & 14.0 & 0.1 & 71.3 & 10.3 & 13.5 \\
\hline Moxidectin & 0.01 & 109.2 & 14.9 & 20.6 & 0.01 & 85.1 & 11.6 & 12.6 \\
\hline & 0.1 & 101.2 & 11.2 & 12.6 & 0.1 & 86.4 & 4.8 & 5.4 \\
\hline Ivermectin & 0.01 & 95.3 & 13.3 & 14.6 & 0.01 & 89.3 & 12.0 & 17.6 \\
\hline & 0.1 & 89.4 & 5.2 & 8.1 & 0.1 & 83.3 & 5.1 & 6.5 \\
\hline Maduramicin & 0.01 & 98.8 & 8.1 & 10.4 & 0.01 & 95.7 & 6.2 & 10.3 \\
\hline & 0.1 & 95.6 & 6.8 & 7.3 & 0.1 & 85.5 & 2.9 & 7.3 \\
\hline Salinomycin & 0.01 & 88.8 & 2.4 & 10.5 & 0.01 & 87.2 & 5.1 & 8.4 \\
\hline & 0.1 & 82.9 & 5.9 & 6.4 & 0.1 & 85.9 & 3.0 & 5.4 \\
\hline Monensin & 0.01 & 89.8 & 4.5 & 9.2 & 0.01 & 98.9 & 11.7 & 19.0 \\
\hline & 0.1 & 90.1 & 7.2 & 8.0 & 0.1 & 87.8 & 3.6 & 13.0 \\
\hline Narasin & 0.01 & 83.8 & 6.0 & 10.0 & 0.01 & 87.1 & 17.2 & 17.7 \\
\hline & 0.1 & 79.6 & 6.5 & 7.4 & 0.1 & 107.3 & 12.3 & 17.4 \\
\hline
\end{tabular}


Table 5. Validated method and LOQ of each analyte

\begin{tabular}{|c|c|c|c|c|c|c|c|c|}
\hline \multirow[b]{2}{*}{ Analyte } & \multicolumn{2}{|c|}{ Bovine muscle } & \multicolumn{2}{|c|}{ Swine muscle } & \multicolumn{2}{|c|}{ Chicken muscle } & \multicolumn{2}{|c|}{ Egg } \\
\hline & $\begin{array}{l}\text { Validated } \\
\text { method }\end{array}$ & $\begin{array}{l}\mathrm{LOQ} \\
(\mu \mathrm{g} / \mathrm{g})\end{array}$ & $\begin{array}{l}\text { Validated } \\
\text { method }\end{array}$ & $\begin{array}{l}\mathrm{LOQ} \\
(\mu \mathrm{g} / \mathrm{g})\end{array}$ & $\begin{array}{l}\text { Validated } \\
\text { method }\end{array}$ & $\begin{array}{l}\mathrm{LOQ} \\
(\mu \mathrm{g} / \mathrm{g})\end{array}$ & $\begin{array}{l}\text { Validated } \\
\text { method }\end{array}$ & $\begin{array}{l}\mathrm{LOQ} \\
(\mu \mathrm{g} / \mathrm{g})\end{array}$ \\
\hline Clopidol & $\mathrm{B}$ & 0.002 & $\mathrm{~B}$ & 0.002 & - & 0.001 & - & 0.001 \\
\hline 5-Hydroxythiabendazole & - & 0.01 & - & 0.005 & - & 0.005 & $\mathrm{~A}$ & 0.005 \\
\hline Levamisole & $\mathrm{A}, \mathrm{B}$ & 0.001 & $\mathrm{~A}, \mathrm{~B}$ & 0.001 & $\mathrm{~A}, \mathrm{~B}$ & 0.001 & $\mathrm{~A}, \mathrm{~B}$ & 0.001 \\
\hline $\begin{array}{l}\text { 5-Propylsulfonyl- } 1 H \text { - } \\
\text { benzimidazole- } 2 \text {-amine }\end{array}$ & B & 0.002 & B & 0.002 & B & 0.002 & B & 0.002 \\
\hline Sulfathiazole & $\mathrm{B}$ & 0.001 & $\mathrm{~A}, \mathrm{~B}$ & 0.001 & $\mathrm{~A}, \mathrm{~B}$ & 0.001 & $\mathrm{~B}$ & 0.001 \\
\hline Trimethoprim & $\mathrm{A}, \mathrm{B}$ & 0.001 & B & 0.001 & B & 0.001 & $\mathrm{~A}, \mathrm{~B}$ & 0.001 \\
\hline Sulfamerazine & $\mathrm{A}, \mathrm{B}$ & 0.001 & $\mathrm{~A}, \mathrm{~B}$ & 0.001 & $\mathrm{~A}, \mathrm{~B}$ & 0.001 & $\mathrm{~A}, \mathrm{~B}$ & 0.001 \\
\hline Ciprofloxacin & - & 0.005 & - & 0.005 & - & 0.005 & - & 0.005 \\
\hline Ormethoprim & $\mathrm{A}, \mathrm{B}$ & 0.001 & $\mathrm{~A}, \mathrm{~B}$ & 0.001 & $\mathrm{~A}, \mathrm{~B}$ & 0.001 & $\mathrm{~A}, \mathrm{~B}$ & 0.001 \\
\hline Enrofloxacin & A & 0.002 & - & 0.002 & $\mathrm{~A}$ & 0.002 & A & 0.002 \\
\hline Thiabendazole & $\mathrm{B}$ & 0.01 & $\mathrm{~B}$ & 0.01 & $\mathrm{~B}$ & 0.01 & $\mathrm{~B}$ & 0.01 \\
\hline Sulfadimidine & $\mathrm{A}, \mathrm{B}$ & 0.001 & $\mathrm{~A}, \mathrm{~B}$ & 0.001 & $\mathrm{~A}, \mathrm{~B}$ & 0.001 & $\mathrm{~A}, \mathrm{~B}$ & 0.001 \\
\hline Sarafloxacin & - & 0.005 & A & 0.005 & - & 0.005 & A & 0.005 \\
\hline Sulfamonomethoxine & $\mathrm{A}, \mathrm{B}$ & 0.001 & $\mathrm{~A}, \mathrm{~B}$ & 0.001 & $\mathrm{~A}, \mathrm{~B}$ & 0.001 & $\mathrm{~A}, \mathrm{~B}$ & 0.001 \\
\hline Sulfamethoxazole & $\mathrm{A}, \mathrm{B}$ & 0.002 & $\mathrm{~A}, \mathrm{~B}$ & 0.002 & $\mathrm{~A}, \mathrm{~B}$ & 0.002 & $\mathrm{~A}, \mathrm{~B}$ & 0.002 \\
\hline Florfenicol & A & 0.005 & $\mathrm{~A}, \mathrm{~B}$ & 0.005 & $\mathrm{~A}, \mathrm{~B}$ & 0.005 & - & 0.005 \\
\hline Oxfendazole & A, B & 0.001 & $\mathrm{~A}, \mathrm{~B}$ & 0.001 & $\mathrm{~A}, \mathrm{~B}$ & 0.001 & $\mathrm{~A}, \mathrm{~B}$ & 0.001 \\
\hline Pyrimethamine & $\mathrm{A}, \mathrm{B}$ & 0.001 & $\mathrm{~A}, \mathrm{~B}$ & 0.001 & $\mathrm{~A}, \mathrm{~B}$ & 0.001 & $\mathrm{~A}, \mathrm{~B}$ & 0.001 \\
\hline Oxolinic acid & A & 0.001 & A & 0.001 & $\mathrm{~A}$ & 0.001 & A & 0.002 \\
\hline Sulfaquinoxaline & $\mathrm{A}, \mathrm{B}$ & 0.001 & $\mathrm{~A}$ & 0.001 & $\mathrm{~A}, \mathrm{~B}$ & 0.001 & - & 0.001 \\
\hline Sulfadimethoxine & $\mathrm{A}, \mathrm{B}$ & 0.001 & $\mathrm{~A}, \mathrm{~B}$ & 0.001 & $\mathrm{~A}, \mathrm{~B}$ & 0.001 & $\mathrm{~B}$ & 0.001 \\
\hline Oxfendazole sulfone & $\mathrm{A}, \mathrm{B}$ & 0.001 & A & 0.001 & $\mathrm{~A}, \mathrm{~B}$ & 0.001 & $\mathrm{~B}$ & 0.001 \\
\hline Nalidixic acid & $\mathrm{A}$ & 0.002 & $\mathrm{~A}$ & 0.002 & $\mathrm{~A}$ & 0.002 & - & 0.002 \\
\hline Flubendazole & $\mathrm{A}, \mathrm{B}$ & 0.001 & $\mathrm{~A}$ & 0.001 & $\mathrm{~A}, \mathrm{~B}$ & 0.001 & - & 0.001 \\
\hline Fenbendazole & $\mathrm{A}, \mathrm{B}$ & 0.001 & A & 0.001 & $\mathrm{~A}, \mathrm{~B}$ & 0.001 & $\mathrm{~A}, \mathrm{~B}$ & 0.001 \\
\hline Nicarbazin & $\mathrm{A}, \mathrm{B}$ & 0.001 & $\mathrm{~A}, \mathrm{~B}$ & 0.001 & B & 0.001 & $\mathrm{~A}, \mathrm{~B}$ & 0.001 \\
\hline Triclabendazole sulfoxide & $\mathrm{A}, \mathrm{B}$ & 0.005 & $\mathrm{~A}, \mathrm{~B}$ & 0.005 & B & 0.005 & $\mathrm{~A}, \mathrm{~B}$ & 0.005 \\
\hline Nequinate & $\mathrm{A}, \mathrm{B}$ & 0.001 & $\mathrm{~A}, \mathrm{~B}$ & 0.001 & $\mathrm{~A}, \mathrm{~B}$ & 0.001 & $\mathrm{~A}, \mathrm{~B}$ & 0.001 \\
\hline Buquinolate & $\mathrm{A}, \mathrm{B}$ & 0.001 & $\mathrm{~A}, \mathrm{~B}$ & 0.001 & $\mathrm{~A}, \mathrm{~B}$ & 0.001 & $\mathrm{~A}, \mathrm{~B}$ & 0.001 \\
\hline Diclazuril & $\mathrm{A}, \mathrm{B}$ & 0.005 & $\mathrm{~A}, \mathrm{~B}$ & 0.005 & $\mathrm{~A}, \mathrm{~B}$ & 0.005 & B & 0.005 \\
\hline Triclabendazole sulfone & $\mathrm{A}, \mathrm{B}$ & 0.001 & $\mathrm{~A}, \mathrm{~B}$ & 0.001 & $\mathrm{~A}, \mathrm{~B}$ & 0.001 & $\mathrm{~A}, \mathrm{~B}$ & 0.001 \\
\hline Triclabendazole & B & 0.002 & B & 0.002 & $\mathrm{~A}, \mathrm{~B}$ & 0.002 & $\mathrm{~A}, \mathrm{~B}$ & 0.002 \\
\hline Decoquinate & $\mathrm{A}$ & 0.001 & $\mathrm{~A}, \mathrm{~B}$ & 0.001 & B & 0.001 & B & 0.001 \\
\hline Eprinomectin & $\mathrm{B}$ & 0.002 & B & 0.002 & $\mathrm{~B}$ & 0.002 & $\mathrm{~B}$ & 0.005 \\
\hline Semduramicin & $\mathrm{B}$ & 0.001 & $\mathrm{~B}$ & 0.001 & $\mathrm{~B}$ & 0.001 & $\mathrm{~B}$ & 0.001 \\
\hline Doramectin & $\mathrm{B}$ & 0.001 & $\mathrm{~B}$ & 0.001 & $\mathrm{~B}$ & 0.002 & $\mathrm{~B}$ & 0.002 \\
\hline Lasalocid & $\mathrm{B}$ & 0.001 & $\mathrm{~B}$ & 0.001 & $\mathrm{~B}$ & 0.001 & $\mathrm{~B}$ & 0.001 \\
\hline Moxidectin & $\mathrm{B}$ & 0.001 & $\mathrm{~B}$ & 0.001 & B & 0.001 & $\mathrm{~B}$ & 0.001 \\
\hline Ivermectin & B & 0.001 & B & 0.001 & $\mathrm{~A}, \mathrm{~B}$ & 0.001 & B & 0.001 \\
\hline Maduramicin & $\mathrm{A}, \mathrm{B}$ & 0.001 & $\mathrm{~A}, \mathrm{~B}$ & 0.001 & $\mathrm{~A}, \mathrm{~B}$ & 0.001 & $\mathrm{~A}, \mathrm{~B}$ & 0.001 \\
\hline Salinomycin & B & 0.001 & B & 0.001 & B & 0.001 & B & 0.001 \\
\hline Monensin & $\mathrm{A}, \mathrm{B}$ & 0.001 & B & 0.001 & B & 0.001 & B & 0.001 \\
\hline Narasin & $\mathrm{A}, \mathrm{B}$ & 0.001 & $\mathrm{~A}, \mathrm{~B}$ & 0.001 & $\mathrm{~A}, \mathrm{~B}$ & 0.001 & $\mathrm{~B}$ & 0.001 \\
\hline
\end{tabular}

were recovered using citrate buffer. It was considered that the lower $\mathrm{pH}$ favored transfer to the acetonitrile layer. Therefore, to allow analysis of as many drugs as possible, the combination of magnesium sulfate, trisodium citrate and sodium chloride was adopted for the buffering.

Second, acidic conditions were studied by comparing no additive, 0.1 and 1 vol\% formic acid as added acids to acetonitrile. The results are shown in Fig. 1. Recoveries of quinolones and fluoroquinolones were increased as the acidity increased, but the mean recoveries of polyethers and macrolides were less than $70 \%$. It was con- sidered that the unionized forms of quinolones increased as the acidity increased, but cleavage of large molecules such as polyethers and macrolides was enhanced ${ }^{23)}$. In egg also, matrix effects were not sufficiently corrected even when using matrix-matched calibration curves, because of the larger amounts of co-extracted matrices derived from the samples. Therefore we used two methods of extraction; method A with an acidic solvent for quinolones, and method B with only acetonitrile, a non-acid solvent, for lipophilic analytes.

Third, the effect of salting-out was studied because the amount of sodium chloride influenced the recovery 
rate and ionization effect from the matrices ${ }^{8,24)}$. Studies used 1.0, 2.0 and $5.0 \mathrm{~g}$ of sodium chloride; however the operation could not be continued in the case of $5.0 \mathrm{~g}$ of sodium chloride because the sample solution formed an emulsion, so only the results for 1.0 and $2.0 \mathrm{~g}$ are shown in Fig. 2. Although there were no differences with more soluble drugs such as sulfonamides, quinolones and fluoroquinolones, the recoveries of polyethers and macrolides were improved.

\section{Method validation}

To validate the method, recovery tests using both method A and method B were performed in accordance with the Japanese guidelines, and the results are shown in Tables 3 and 4. Quantification values were obtained from matrix-matched calibration curves where the correlation coefficient $\left(r^{2}\right)$ was over 0.999. In method A, the number of analytes that satisfied the criteria of the guidelines was 27 analytes out of 43 in bovine and swine muscle, 26 in chicken muscle and 21 in egg. In method B the results were 35 analytes in bovine muscle, 33 in swine muscle and egg, and 36 in chicken muscle.

Quinolones and fluoroquinolones showed higher accuracy in method A, acidic extraction. However, some fluoroquinolones were not sufficiently recovered even using method A because they show amphoteric characteristics, which made them more soluble in the water layer than in the acetonitrile layer. In contrast, polyethers and macrolides were well validated using method B. Highly hydrophilic analytes such as 5-hydroxythiabendazole and 5-propylsulfonyl-1H-benzimidazole-2-amine (both metabolites) were difficult to recover in either method because they are highly soluble in water, and were not transferred to the organic layer.

Selectivities were confirmed by analyzing blank samples, and there were no interfering peaks at the same retention times as the target analytes.

LOQs are shown in Table 5, and they were less than the MRLs.

\section{Conclusion}

In this study, new methods for rapid analysis of multiclass residual veterinary drugs in livestock products were developed, and were validated according to the Japanese validation guidelines. Analytical methods were developed using LC-MS/MS and results were achieved in only $18 \mathrm{~min}$ to analyze 43 analytes simultaneously. Sample preparation processes were developed by modifying the QuEChERS approach and using two extraction solvents; method A which included 1 vol\% formic acid in acetonitrile obtained higher recoveries of quinolones, and method $\mathrm{B}$ which did not include acid was used for polyethers and macrolides. Using these methods, the number of analytes that were validated in accordance with the Japanese validation guidelines was 40 analytes out of 43 in bovine and swine muscle, 39 in chicken muscle, and 37 in egg.

\section{References}

1) Van den Bogaard, A. E., Stobberingh, E. E. Epidemiology of resistance to antibiotics. Links between animals and humans. Int. J. Antimicrob. Agent, 14, 327-335 (2000).

2) Noguchi, S., Terada, H., Tamura, Y. Simultaneous determination of veterinary drugs in livestock foods and seafoods using liquid chromatography/tandem mass spectrometry. J. Food Hyg. Soc. Japan, 49, 177-188 (2008).

3) Kajita, H., Hatakeyama, E. Simultaneous determination of residual veterinary drugs in livestock products and fish by liquid chromatography with tandem mass spectrometry. J. Food Hyg. Soc. Japan, 49, 381-389 (2008).

4) Inoue, K., Yoshimi, Y., Hino, T., Oka, H. LC/ESI-MS/MS method for the simultaneous determination of macrocyclic lactone parasiticides in livestock products and fish. J. Food Hyg. Soc. Japan, 51, 1-9 (2010).

5) Heller, D. N., Nochetto, C. B., Rummel, N. G., Thomas, M. H. Development of multiclass methods for drug residues in eggs: hydrophilic solid-phase extraction cleanup and liquid chromatography/tandem mass spectrometry analysis of tetracycline, fluoroquinolone, sulfonamide, and beta-lactam residues. J. Agric. Food Chem., 54, 5267-5278 (2006)

6) Yamada, R., Kozono, M., Ohmori, T., Morimatsu, F., Kitayama, M. Simultaneous determination of residual veterinary drugs in bovine, porcine, and chicken muscle using liquid chromatography coupled with electrospray ionization tandem mass spectrometry. Biosci. Biotechnol. Biochem., 70, 54-65 (2006).

7) Kaufmann, A., Butcher, P., Maden, K., Widmer, M. Quantitative multiresidue method for about 100 veterinary drugs in different meat matrices by sub 2-micron particulate high-performance liquid chromatography coupled to time of flight mass spectrometry. J. Chromatogr. A, 1194, 66-79 (2008).

8) Anastassiades, M., Lehotay, S. J., Stajnbaher, D., Schenck, F. J. Fast and easy multiresidue method employing acetonitrile extraction/partitioning and "dispersive solid-phase extraction" for the determination of pesticide residues in produce. J. AOAC Int., 86, 412-431 (2003).

9) Lehotay, S. J., Mastovska, K., Lightfield, A. R. Use of buffering and other means to improve results of problematic pesticides in a fast and easy method for residue analysis of fruits and vegetables. J. AOAC Int., 88, 615-629 (2005)

10) Lehotay, S. J. et al. Determination of pesticide residues in foods by acetonitrile extraction and partitioning with magnesium sulfate: collaborative study. J. AOAC Int., 90, 485-520 (2007).

11) Paya, P., Anastassiades, M., Mack, D., Sigalova, I., Tasdelen, B., Oliva, J., Barba, A. Analysis of pesticide residues using the Quick Easy Cheap Effective Rugged and Safe (QuEChERS) pesticide multiresidue method in combination with gas and liquid chromatography and tandem mass spectrometric detection. Anal. Bioanal. Chem., 389, 1697-1714 (2007)

12) Lazartigues, A., Wiest, L., Baudot, R., Thomas, M., Feidt, C., Cren-Olive, C. Multiresidue method to quantify pesticides in fish muscle by QuEChERS-based extraction and LC-MS/MS. Anal. Bioanal. Chem., 400, 2185-2193 (2011)

13) Jiang, Y., Li, X., Xu, J., Pan, C., Zhang, J., Niu, W. Multiresidue method for the determination of 77 pesticides in wine using QuEChERS sample preparation and gas 
chromatography with mass spectrometry. Food Addit. Contam. Part A, 26, 859-866 (2009).

14) Chung, S. W., Chan, B. T. Validation and use of a fast sample preparation method and liquid chromatographytandem mass spectrometry in analysis of ultra-trace levels of 98 organophosphorus pesticide and carbamate residues in a total diet study involving diversified food types. J. Chromatogr. A, 1217, 4815-4824 (2010).

15) Gilbert-Lopez, B., Garcia-Reyes, J. F., Lozano, A., Fernandez-Alba, A. G., Molina-Diaz, A. Large-scale pesticide testing in olives by liquid chromatography-electrospray tandem mass spectrometry using two sample preparation methods based on matrix solid-phase dispersion and QuEChERS. J. Chromatogr. A, 1217, 6022-6035 (2010).

16) Martinez Vidal, J. L., Frenich, A. G., Aguilera-Luiz, M. M., Romero-Gonzalez, R. Development of fast screening methods for the analysis of veterinary drug residues in milk by liquid chromatography-triple quadrupole mass spectrometry. Anal. Bioanal. Chem., 397, 2777-2790 (2010).

17) Frenich, A. G., Aguilera-Luiz, M. M., Martinez Vidal, J. L., Romero-Gonzalez, R. Comparison of several extraction techniques for multiclass analysis of veterinary drugs in eggs using ultra-high pressure liquid chromatography-tandem mass spectrometry. Anal. Chim. Acta, 661, 150-160 (2010).

18) Stubbings, G., Bigwood, T. The development and validation of a multiclass liquid chromatography tandem mass spectrometry (LC-MS/MS) procedure for the determina- tion of veterinary drug residues in animal tissue using a QuEChERS (QUick, Easy, CHeap, Effective, Rugged and Safe) approach. Anal Chim. Acta, 637, 68-78 (2009).

19) Kinsella, B., Whelan, M., Cantwell, H., McCormack, M., Furey, A., Lehotay, S. J., Danaher, M. A dual validation approach to detect anthelmintic residues in bovine liver over an extended concentration range. Talanta, 83, 1424 (2010).

20) Blasco, C., Masia, A., Morillas, F. G., Pico, Y. Comparison of the effectiveness of recent extraction procedures for antibiotic residues in bovine muscle tissues. J. AOAC Int., 94, 991-1003 (2011).

21) Lombardo-Agui, M., Gamiz-Gracia, L., Cruces-Blanco, C., Garcia-Campana, A. M. Comparison of different sample treatments for the analysis of quinolones in milk by capillary-liquid chromatography with laser induced fluorescence detection. J. Chromatogr. A, 1218, 4966-4971 (2011).

22) Inoue, K., Yoshimi, Y., Hino, T., Oka, H. Simultaneous determination of avermectins in bovine tissues by LCMS/MS. J. Sep. Sci., 32, 3596-3602 (2009).

23) Wells, J. L., Bordner, J., Bowles, P., McFarland, J. W. Novel degradation products from the treatment of salinomycin and narasin with formic acid. J. Med. Chem., 31, 274-276 (1988).

24) Xia, X., Li, X., Shen, J., Jiang, H. Determination of nitroimidazole residues in porcine urine by liquid chromatography/tandem mass spectrometry. J. AOAC Int., 89, 9499 (2006). 